\title{
The Equivalence Problem for Letter-to-Letter Bottom-up Tree Transducers is Solvable*
}

\author{
Yves ANDRE and Francis BOSSUT \\ L.I.F.L., U.R.A. 369 C.N.R.S. \\ University of Lille 1, 59655 Villeneuve d'Ascq Cedex. France. \\ e-mail:\{andre, bossut\}@ liff.lifl.fr
}

\begin{abstract}
Letter-to-letter bottom-up tree transducers are investigated in this paper. With an encoding of the so defined tree transformations into relabelings, we establish the decidability of equivalence for this class of tree transducers. Some extensions are next given.
\end{abstract}

\section{Introduction}

From a general point of view, tree transducers investigate computations on structured objects. Consider a concrete algorithm $A$ taking terms from $T_{\Sigma}$ as input and producing terms of $T_{\Delta}$, where $\Sigma$ and $\Delta$ are finite sets of operators. Abstracting from the meaning of the operators, $A$ turns into a symbolic algorithm which is a tree transducer transforming elements of $T_{\Sigma}$ into elements of $T_{\Delta}$.

Finite state tree transducers, which are a generalization of Generalized Sequential Machines in the word case, were introduced by Rounds and Thatcher [13, 15] in order to give an algebraic formalism for the theory of syntax-directed translation. Let us give some examples. In compiler construction finite state transducers can be used to express simple transformations of abstract syntactical trees. Attribute grammars with only synthesized attributes correspond closely to deterministic top-down finite state transducers.

For the description of a larger class of operations on tree more subtle devices are needed as macro tree transducers [8]; general operations on trees (primitive recursive functions) can be defined in term of modular tree transducers [9].

For the formal language point of view the question arises whether or not results obtained for such transformations in the word case can be transferred in the tree case, especially decidability of equivalence (i) and closure properties with respect to composition (ii). The situation is more complex because we have to distinguish two main classes of finite state tree transducers: top-down transducers which process the input trees from the root to the leaves and bottom-up transducers for which, on the contrary, the computations begin at the leaves and finish at the root (a comparison between these two classes can be found in [6]). As noteworthy characteristics in the bottom-up case, let us point out the fact that since some variables can be missing, the image of a correctly parsed subtree can be deleted.

\footnotetext{
* Supported in part by the PRC-GDR "Mathématiques et Informatique" and the Basic Research Working Group ESPRIT 6317 ASMICS II
} 
(i) Two transducers are called equivalent if they define the same translation. It is well-known that equivalence is, in general, undecidable for non-deterministic tree transducers and that it is decidable for deterministic transducers, in the bottomup case ( $Z$. Zachar [16]) as in the top-down one ( $Z$. Esik [10]). More recently, H. Seidl established the decidability of equivalence for finite-valued bottom-up transducers [14].

(ii) The class of top-down tree transformation is not closed under composition. In 1977, J. Engelfriet defined the notion of top-down tree transducer with regular look-ahead in order to get nice properties with respect to composition [7]. These top-down transducers are able to inspect a subtree before deleting it. Moreover, he showed that the class of top-down tree transducers with regular look-ahead is a subset of the class of transformation realized by composition of bottom-up and top-down transducers. Many classes of top-down tree transducers with lookahead have been studied. Let us quote the works of Z. Fülöp and S. Vágvölgyi ([11] for instance).

This paper is part of a study of letter-to-letter tree transducers. Informally, trees which appear in the rules of these transducers are reduced to one letter in the right-hand side as in the left-hand one. By means of an example, we first illustrate this kind of transducers.

Example 1 Let us consider some rules ${ }^{1}$ of a bottom-up letter-to-letter transducer, which realizes simplifications of formulae of propositional logic

$$
\begin{array}{rcc}
1 \rightarrow q_{1}(1) & \neg\left(q_{\perp}(x)\right) \rightarrow q_{\perp}(\neg(x)) & \wedge\left(q_{0}(x), q_{\perp}(y)\right) \rightarrow q_{0}(0) \\
0 \rightarrow q_{0}(0) & \wedge\left(q_{\perp}(x), q_{\perp}(y)\right) \rightarrow q_{\perp}(\wedge(x, y)) & \wedge\left(q_{\perp}(x), q_{0}(y)\right) \rightarrow q_{0}(0) \\
v \rightarrow q_{\perp}(v)^{2} & \wedge\left(q_{1}(x), q_{\perp}(y)\right) \rightarrow q_{\perp}(y) & \wedge\left(q_{0}(x), q_{1}(y)\right) \rightarrow q_{0}(0) \\
\neg\left(q_{1}(x)\right) \rightarrow q_{0}(0) & \wedge\left(q_{\perp}(x), q_{1}(y)\right) \rightarrow q_{\perp}(x) & \wedge\left(q_{1}(x), q_{0}(y)\right) \rightarrow q_{0}(0) \\
\neg\left(q_{0}(x)\right) \rightarrow q_{1}(1) & \wedge\left(q_{1}(x), q_{1}(y)\right) \rightarrow q_{1}(1) & \wedge\left(q_{0}(x), q_{0}(y)\right) \rightarrow q_{0}(0)
\end{array}
$$

This transducer is letter-to-letter, deterministic and linear but it is deleting. The

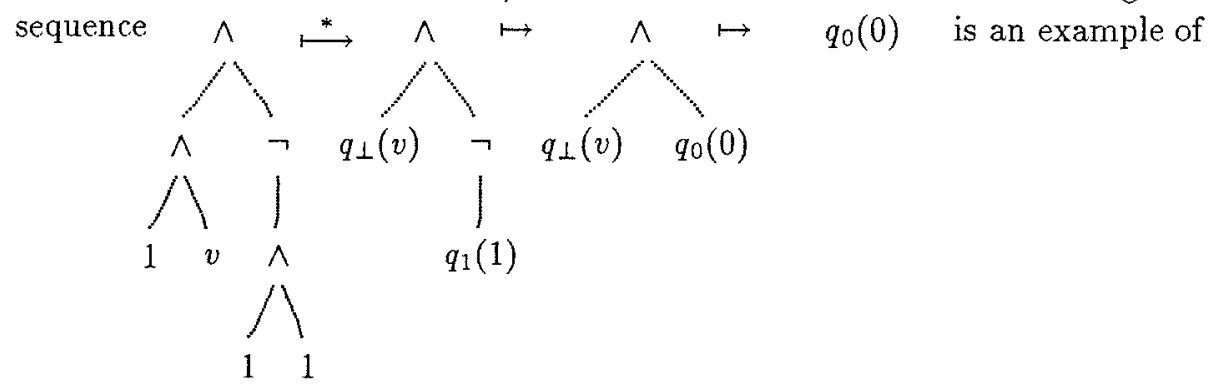

computations.

\footnotetext{
1 The reader can easily find the rules involving the operator "or" (V).

2 for any atomic proposition symbol $v$
} 
In previous works $[1,2,3]$, we established the decidability of equivalence for linear transducers and for non-deleting transducers in the top-down case. Here, we prove the decidability of equivalence for letter-to-letter bottom-up transducers. As a corollary of this result and previous ones, we can deduce the decidability of equivalence for some subclasses of letter-to-letter transducers with regular lookahead.

Some authors (Coquidé et al. [5], for instance) showed that, in some particular cases, one can construct a bottom-up tree pushdown automata computing normal forms of ground terms with respect to a rewrite system. As one may view a bottom-up tree pushdown automaton as a transducer that outputs its pushdown, a subject of further investigation should be to study letter-to-letter rewrite systems; especially, the properties of the associated letter-to-letter transducer.

\section{Basic Definitions and Properties}

In this section, we just recall definitions and properties used in the sequel. We refer the reader to $[6,7]$ for tree transducers and to [12] for tree automata.

A ranked alphabet is a pair $(\Sigma, \rho)$ where $\Sigma$ is a finite alphabet and $\rho$ is a mapping from $\Sigma$ to $\mathbb{N}$. Usually, we will write $\Sigma$ for short. For any $\sigma$ of $\Sigma, \rho(\sigma)$ is called the rank of $\sigma$. For any integer $n, \Sigma_{n}$ denotes the subset of $\Sigma$ of letters of rank $n$. For any $k \geq 1, X_{k}$ denotes the set of variables $\left\{x_{1}, . ., x_{k}\right\}$.

(iiven a ranked alphabet $\Sigma$ and a denumerable set $X$ of variables, $T_{\Sigma}(X)$ denotes the set of all terms (trees) over $\Sigma$ and indexed by $X$. In the particular case of an empty set of variables, we will write $T_{\Sigma}$ instead of $T_{\Sigma}(\emptyset)$. A tree $t_{o}\left(x_{1}, \ldots, x_{n}\right)$ is a prefix of a term $t$ if there exist $t_{1}, . ., t_{n}$ such that $t=t_{o}\left(t_{1}, . ., t_{n}\right)$. For any tree $t$ of $T_{\Sigma}(X)$, the height of $t$, denoted by $\pi(t)$, is defined by $\pi(t)=0$ if $t \in \Sigma_{0}$ or $t \in X$ and $\pi(t)=1+\max \left\{\pi\left(t_{1}\right), . ., \pi\left(t_{n}\right)\right\}$ if $t=\sigma\left(t_{1}, . ., t_{n}\right)$.

For any $p \in \mathbb{N},[p]$ denotes the set $\{1, \ldots, p\}$. A torsion $\theta$ from $[p]$ to $[q]$ is a mapping from $[p]$ to $[q]$. We denote it by $\langle q ; \theta(1), . ., \theta(p)\rangle$. By $i d_{[n]}$ we denote the identity mapping on $[n]$.

A finite state bottom-up tree transducer is a 5-tuple $B=\langle\Sigma, \Delta, Q, F, R>$ where $\Sigma$ and $\Delta$ are ranked alphabets of respectively input and output symbols, $Q$ is a finite set of unary symbols called states, $F$ is the subset of $Q$ of final states and $R$ is a finite set of rules of the form $\sigma\left(q_{1}\left(x_{1}\right), \ldots, q_{n}\left(x_{n}\right)\right) \mapsto q(t)$ with $q, q_{1}, . ., q_{n} \in Q$, $\sigma \in \Sigma_{n}$ and $t \in T_{\Delta}\left(X_{n}\right)$ or of the form $\sigma \mapsto q(t)$ with $\sigma \in \Sigma_{0}$ and $t \in T_{\Delta}$. In this paper, these rules will be denoted by $\sigma\left(q_{1}\left(x_{1}\right), \ldots, q_{n}\left(x_{n}\right)\right) \rightarrow q\left(\delta\left(x_{\theta(1)}, \ldots, x_{\theta(p)}\right)\right)$ with $\delta \in T_{\Delta}\left(X_{n}\right)$ and $\theta$ torsion from $[p]$ to $[n]$ (if $n=0$ we write $\sigma \rightarrow q(\delta)$ ). So torsions $\theta$ will express permutations, duplications or deletions of subtrees. A transducer is letter-to-letter if, for every rule, $\delta$ belongs to $\Delta$.

The rules define patterns for rewriting trees, so we write $t \mapsto u$ if $t$ is rewritten in $u$ in one step. By $\stackrel{*}{\longmapsto}$ we denote the reflexive and transitive closure of $\mapsto$. A sequence of rewriting steps $t \stackrel{*}{\longmapsto} q(u)$ is called a computation. 
Let $B$ be a bottom-up transducer and $\mathcal{D}$ be a computation on a term $t=$ $\sigma\left(t_{1}, . ., t_{m}\right), \quad \mathcal{D}: \sigma\left(t_{1}, . ., t_{m}\right) \stackrel{*}{\longmapsto} \sigma\left(q_{1}\left(u_{1}\right), . ., q_{m}\left(u_{m}\right)\right) \mapsto q\left(\delta\left(u_{\theta(1)}, . ., u_{\theta(n)}\right)\right)$. The triple $(\sigma, \delta, \theta)$ is called the final transformation.

Let $B$ be a bottom-up transducer, $q$ and $E$ be respectively a state and a set of states of $B$ and let $(\sigma, \delta, \theta)$ be a final transformation. $\widehat{B}_{q}$ denotes the set $\left\{(t, u) \in T_{\Sigma} \times T_{\Delta} \mid t \stackrel{*}{\longmapsto} q(u)\right\}, \widehat{B}_{E}=\cup_{q \in E} \widehat{B}_{q}$ and we denote by $\widehat{B}_{q_{(\sigma, \delta, \theta)}}$ the set $\left\{(t, u) \mid t=\sigma\left(t_{1}, . ., t_{m}\right)\right.$ and $t \stackrel{*}{\longmapsto} B \sigma\left(q_{1}\left(u_{1}\right), . ., q_{m}\left(u_{m}\right) \mapsto q\left(\delta\left(u_{\theta(1)}, . ., u_{\theta(n)}\right)\right)=\right.$ $q(u)\}$. Obviously $\widehat{B}_{E_{(\sigma, \delta, \theta)}}=\cup_{q \in E} \widehat{B}_{q_{(\sigma, \delta, \theta)}}$. Finally we denote by $\widehat{B}$ the tree transformation associated with $B: \widehat{B}=\widehat{B}_{F}$ (where $F$ is the set of final states).

Two sets of states $E$ and $F$ of a transducer $B$ are globally equivalent if and only if $\hat{B}_{E}=\widehat{B}_{F}$. Two transducers $B$ and $B^{\prime}$ are equivalent if and only if $\widehat{B}=\widehat{B}^{\prime}$. The domain of a tree transformation $\widehat{B}$, denoted by $\operatorname{dom}(\widehat{B})$, is the regular language $\left\{t \in T_{\Sigma} / \exists u \in T_{\Delta},(t, u) \in \widehat{B}\right\}$. The range of a tree transformation $\widehat{B}$, denoted by range $(\widehat{B})$, is the set $\left\{u \in T_{\Delta} / \exists t \in T_{\Sigma},(t, u) \in \widehat{B}\right\}$. A tree transformation $\widehat{B}$ is of finite range if its range is finite. We extend the last definition to any subset of a tree transformation: a subset $S$ of a tree transformation $\widehat{B}$ is of finite range if the set $\{u \mid \exists t$ such that $(t, u) \in S\}$ is finite.

A state $q$ of a transducer $B$ is infinitary if and only if $\widehat{B}_{q}$ is of infinite range. A transducer all states of which are infinitary is said to be infinitary. A bottom-up tree transducer is deterministic if and only if the set of final states is a singleton and there are no two rules with the same left-hand side.

A transducer is linear (respectively non-deleting, torsion-free) if and only if for each rule the torsion $\theta$ is injective (respectively surjective, the identity mapping). A torsion-free letter-to-letter transducer is also called a relabeling.

Property 1. For any letter-to-letter tree transducer $B$, for any couple of trees $(t, u)$ of $\widehat{B}$, we have $\pi(t)=\pi(u)$ if $B$ is non-deleting and $\pi(t) \geq \pi(u)$ in the other cases.

We denote by B-LL the class of all bottom-up letter-to-letter tree transducers. By LB-LL (respectively NdB-LL, B-LAB), we will denote the subclass of $\mathbf{B}-$ LL of Linear transducers (resp. Non-deleting transducers, relabelings).

Symmetrically, we define a top-down tree transducer as a 5 -tuple $T=<\Sigma, \Delta, Q, I$, $R>$ where $I$ is the subset of $Q$ of initial states and $R$ is a finite set of rules of the form $q\left(\sigma\left(x_{1}, \ldots, x_{n}\right)\right) \rightarrow \delta\left(q_{1}\left(x_{\theta(1)}\right), . ., q_{p}\left(x_{\theta(p)}\right)\right)$ or of the form $q(\sigma) \rightarrow \delta$.

T-LL is the class of all top-down letter-to-letter tree transducers.

A top-down tree transducer with regular look-ahead is a 5 -tuple $T=<\Sigma, \triangle, Q, I$, $R>$ where $\Sigma, \Delta, Q$ and $I$ are defined as for classical top-down transducers and $R$ is a finite set of rules of the form $t \rightarrow t^{\prime}, D$ where $t \rightarrow t^{\prime}$ is a classical rule and $D$ is a mapping from the set of variables occurring in $t$ to recognizable subsets of $T_{\Sigma}$ (more details can be found in [7]). 
The class of all top-down transducers with regular look-ahead is denoted by $\mathbf{T}^{\mathbf{R}}$-FST (for Finite State Transformation). Here, we will use $\mathbf{T}^{\mathbf{R}}$-LL for the subclass of $\mathbf{T}^{\mathbf{R}}$-FST of letter-to-letter transducers.

Remark For easier exposition, we will restrict ourselves to letters of rank at most 2. It is the typical case from which constructions and results to be discussed below are easily transferred to the general situation (with adaptations in the statement of some lemmas).

\section{Equivalence of bottom-up transducers}

In this section, we show that the equivalence problem for letter-to-letter bottomup tree transducers can be reduced to the equivalence of relabelings.

The main problem, which is illustrated by the following example, is that even if two transducers are equivalent, for some trees, different torsions must be necessarily applied in the computations.

Example 2 Let $B_{1}$ and $B_{2}$ be the transducers defined by the following rules:

$$
\begin{aligned}
& B_{1}: \bar{a} \rightarrow q_{1}(e) \\
& \bar{a} \rightarrow q_{2}(e) \\
& \bar{a} \rightarrow q_{1}^{\prime}(\bar{\alpha}) \\
& a\left(q_{1}^{\prime \prime}(x)\right) \rightarrow q_{1}(e) \\
& a\left(q_{1}^{\prime}(x)\right) \rightarrow q_{1}^{\prime \prime}(\alpha(x)) \\
& \sigma\left(q_{1}\left(x_{1}\right), q_{2}\left(x_{2}\right)\right) \rightarrow q\left(\delta\left(x_{1}, x_{2}\right)\right) \\
& \bar{a} \rightarrow q_{2}^{\prime}(\bar{\beta}) \\
& a\left(q_{2}^{\prime}(x)\right) \rightarrow q_{2}(e) \\
& a\left(q_{1}^{\prime \prime}(x)\right) \rightarrow q_{1}^{\prime}(\alpha(x)) \quad a\left(q_{2}^{\prime}(x)\right) \rightarrow q_{2}^{\prime}(\beta(x))
\end{aligned}
$$

The transducer $B_{2}$ is defined in the same way. The only difference is that we have $\sigma\left(q_{1}\left(x_{1}\right), q_{2}\left(x_{2}\right)\right) \rightarrow k\left(\delta\left(x_{2}, x_{1}\right)\right)$ instead of the rule $\sigma\left(q_{1}\left(x_{1}\right), q_{2}\left(x_{2}\right)\right) \rightarrow$ $q\left(\delta\left(x_{1}, x_{2}\right)\right)$. We have $\widehat{B}_{1_{q}}=\widehat{B}_{2_{k}}=\left\{\left(\sigma\left(a^{2 n}(\bar{a}), a^{m}(\bar{a})\right), \delta(e, e)\right) \mid n, m \in \mathbb{I N}\right\}$. But, different final transformations are applied in the computations reaching state $q$ and those reaching state $k$. We first show that this phenomenon only occurs for output trees of "bounded depth".

\subsection{Final transformations}

For technical reasons, only infinitary transducers are investigated in the first lemma. Next, a generalization is given.

Lemma 1. Let $q$ and $k$ be two equivalent states of an infinitary transducer $B$. For any final transformation $(\sigma, \delta, \theta)$, the difference $\widehat{B}_{q_{(\sigma, \delta, \theta)}}-\widehat{B}_{k_{(\sigma, \delta, \theta)}}$ is of finite range.

Proof: Let $\sigma$ be a letter of rank 2. According to the rank of $\delta$ we will distinguish two cases: $\delta$ of rank 2 and $\delta$ of rank $1 . \theta$ is a mapping from $\rho(\delta)$ to [2].

First case: $\delta$ is a letter of rank 2 .

First, assume that $\theta$ is the identity mapping on [2] and so the rules are of the 
form $\sigma\left(q_{i}\left(x_{1}\right), q_{i}^{\prime}\left(x_{2}\right)\right) \rightarrow q\left(\delta\left(x_{1}, x_{2}\right)\right)$ (the case $\theta=<2 ; 2,1>$ is quite similar).

Let $n$ be the number of rules of the form $\sigma\left(q_{i}\left(x_{1}\right), q_{i}^{\prime}\left(x_{2}\right)\right) \rightarrow q\left(\delta\left(x_{1}, x_{2}\right)\right)$, and $m$ be the number of rules of the form $\sigma\left(k_{i}\left(x_{1}\right), k_{i}^{\prime}\left(x_{2}\right)\right) \rightarrow k\left(\delta\left(x_{1}, x_{2}\right)\right)$.

The difference $\widehat{B}_{q_{(\sigma, \delta, \theta)}}-\widehat{B}_{k_{(\sigma, \delta, \theta)}}$ is considered.

We denote by $\mathcal{Q}$ the set $\bigcup_{i \in[n]} \widehat{B}_{q_{z}} \times \widehat{B}_{q_{z}^{\prime}}$ and similarly $\mathcal{K}=\bigcup_{j \in[m]} \widehat{B}_{k_{j}} \times \widehat{B}_{k_{2}^{\prime}}$.

So we have $\left(\sigma\left(u, u^{\prime}\right), \delta\left(v, v^{\prime}\right)\right) \in \widehat{B}_{q_{(\sigma, \delta, \theta)}}-\widehat{B}_{k_{(\sigma, \delta, \theta)}} \Leftrightarrow\left((u, v),\left(u^{\prime}, v^{\prime}\right)\right) \in \mathcal{Q}-\mathcal{K}$.

Elementary properties of the theory of sets allow us to express $\mathcal{Q}-\mathcal{K}$ in terms of $\widehat{B}_{q_{2}}, \widehat{B}_{q_{2}^{\prime}}, \widehat{B}_{k_{3}}, \widehat{B}_{k_{3}^{\prime}}, \bar{B}_{k_{j}}$ and $\bar{B}_{k_{j}^{\prime}}\left(\bar{B}_{k_{3}}\right.$ is the complementation of $\widehat{B}_{k_{3}}$ in $\left.T_{\Sigma} \times T_{\Delta}\right)$.

$$
\begin{aligned}
\mathcal{Q}-\mathcal{K} & =\left(\bigcup_{i \in[n]} \hat{B}_{q_{\mathrm{i}}} \times \hat{B}_{q_{i}^{\prime}}\right)-\left(\bigcup_{\jmath \in[m]} \hat{B}_{k_{\jmath}} \times \hat{B}_{k_{\jmath}^{\prime}}\right) \\
& =\bigcup_{i \in[n]}\left(\bigcup_{J \subseteq[m]}\left(\hat{B}_{q_{\mathrm{t}}} \cap\left(\bigcap_{j \in J}\left(\bar{B}_{k_{\jmath}}\right)\right)\right) \times\left(\widehat{B}_{q_{2}^{\prime}} \cap\left(\bigcap_{l \in[m]-J}\left(\bar{B}_{k_{l}^{\prime}}\right)\right)\right)\right) .
\end{aligned}
$$

Assume that the difference $\widehat{B}_{q_{(\sigma, \delta, \theta)}}-\widehat{B}_{k_{(\sigma, \delta, \theta)}}$ is not of finite range. In this case, there would exist at least $i \in[n]$ and $J \subseteq[m]$ such that $\widehat{B}_{q_{2}} \cap\left(\bigcap_{j \in J}\left(\bar{B}_{k_{j}}\right)\right)$ or $\widehat{B}_{q_{2}^{\prime}} \cap\left(\bigcap_{l \in[m]-J}\left(\bar{B}_{k_{1}^{\prime}}\right)\right)$ is not of finite range. Suppose now that $\widehat{B}_{q_{2}} \cap\left(\bigcap_{j \in J}\left(\bar{B}_{k_{3}}\right)\right)$ is not of finite range.

Thus for every couple $\left(u^{\prime}, v^{\prime}\right)$ in $\widehat{B}_{q_{1}^{\prime}} \cap\left(\bigcap_{l \in[m]-J}\left(\bar{B}_{k_{l}^{\prime}}\right)\right)$, we could find a couple $(u, v)$ of $\widehat{B}_{q_{v}} \cap\left(\bigcap_{j \in J}\left(\bar{B}_{k_{j}}\right)\right)$ such that $\pi(v)>\pi\left(u^{\prime}\right)$ (B is an infinitary transducer) that is to say a couple $\left(\sigma\left(u, u^{\prime}\right), \delta\left(v, v^{\prime}\right)\right) \in \widehat{B}_{q_{(\sigma, \delta, \theta)}}-\widehat{B}_{k_{(\sigma, \delta, \theta)}}$ with $\pi(v)>\pi\left(u^{\prime}\right)$. Because $q$ and $k$ are equivalent states, there would exist another torsion $\mu(\mu \neq \theta)$ such that $\left(\sigma\left(u, u^{\prime}\right), \delta\left(v, v^{\prime}\right)\right) \in \widehat{B}_{k(\sigma, \delta, \mu)}$. The torsion $\mu$ should be of one of the following forms: $\mu=\langle 2 ; 2,1>, \mu=<2 ; 1,1>$ or $\mu=<2 ; 2,2>$. Each case leads to a contradiction.

If $\mu=\left\langle 2 ; 2,1>\right.$, for instance, we should have $\sigma\left(u, u^{\prime}\right) \stackrel{*}{\longmapsto} \sigma\left(k_{j}\left(v^{\prime}\right), k_{j}^{\prime}(v)\right) \mapsto$ $k\left(\delta\left(v, v^{\prime}\right)\right)$. Such computations are impossible because we should have $\pi(v) \leq$ $\pi\left(u^{\prime}\right)$ (property 1) when we chose $\pi(v)>\pi\left(u^{\prime}\right)$.

The same result is obtained for $\theta=\langle 2 ; 1,1>$ or $\theta=\langle 2 ; 2,2\rangle$ and so we conclude that the difference $\hat{B}_{q_{(\sigma, \delta, \theta)}}-\widehat{B}_{k_{(\sigma, \delta, \theta)}}$ is of finite range when $\sigma$ and $\delta$ are of rank 2 .

Second case: $\delta$ is of rank 1 .

The rules are now of the form $\sigma\left(q_{i}\left(x_{1}\right), q_{i}^{\prime}\left(x_{2}\right)\right) \rightarrow q\left(\delta\left(x_{\theta(1)}\right)\right)$ where $\theta$ is either $\langle 2 ; 1\rangle$ or $\langle 2 ; 2\rangle$. Let $\theta=\langle 2 ; 1\rangle$; the other case is quite similar.

Let us suppose that $\widehat{B}_{q_{(\sigma, \delta, \theta)}}-\widehat{B}_{k_{(\sigma, \delta, \theta)}}$ is not of finite range. Then there would exist an infinite number of trees $v$ such that we can find $u$ and $u^{\prime}$ in $T_{\Sigma}$ with $\left(\sigma\left(u, u^{\prime}\right), \delta(v)\right) \notin \widehat{B}_{k_{(\sigma, \delta, \theta)}}$. In particular, there would exist $v$ and $u^{\prime}$ such that $\pi(v)>\pi\left(u^{\prime}\right)$. 
Now $q$ and $k$ are equivalent states and so there exists an other torsion $\mu$ such that $\left(\sigma\left(u, u^{\prime}\right), \delta(v)\right) \in \widehat{B}_{k_{(\sigma, \delta, \mu)}}$. Here we would have $\mu=<2 ; 2>$ and $\sigma\left(u, u^{\prime}\right) \stackrel{*}{\longmapsto}$ $\sigma\left(k_{i}(w), k_{i}^{\prime}(v)\right) \mapsto k(\delta(v))$. Such computations cannot be realized because we should have $\pi(v)>\pi\left(u^{\prime}\right)$ and $\pi(v) \leq \pi\left(u^{\prime}\right)$.

Therefore, in all cases, for any final transformation $(\sigma, \delta, \theta)$ the difference $\widehat{B}_{q_{(\sigma, \delta, \theta)}}$ $\widehat{B}_{k_{(\sigma, \delta, \theta)}}$ is of finite range.

Corollary 1. Let $B$ be a letter-to-letter bottom-up transducer. There exists a natural number, denoted by $\Lambda$, such that for any globally equivalent sets of states $E$ and $F$, for any final transformation $(\sigma, \delta, \theta)$, as soon as $\pi(u)>\Lambda$, we have $(t, u) \in \widehat{B}_{E_{(\sigma, \delta, \theta)}} \Leftrightarrow(t, u) \in \widehat{B}_{F_{(\sigma, \delta, \theta)}}$.

Proof: A result analogous to lemma 1 is obtained when we consider globally equivalent sets of states $E$ and $F$ (instead of equivalent states) of an infinitary transducer: for any final transformation $(\sigma, \delta, \theta)$, the difference $\widehat{B}_{E_{(\sigma, \delta, \theta)}}-\widehat{B}_{F_{(\sigma, \delta, \theta)}}$ is of finite range and so, obviously, there exists a natural number $\lambda_{(E, F),(\sigma, \delta, \theta)}$, such that as soon as $\pi(u)>\lambda_{(E, F),(\sigma, \delta, \theta)}$, we have $(t, u) \in \widehat{B}_{E_{(\sigma, \delta, \theta)}} \Leftrightarrow(t, u) \in$ $\widehat{B}_{F_{(\sigma, \delta, \theta)}}$.

To extend this result to a transducer some states of which are finitary, we take $\lambda_{(E, F),(\sigma, \delta, \theta)}$ greater than the number of finitary states of this transducer. Now let $\lambda$ be an upper-bound for these integers $\lambda_{(E, F),(\sigma, \delta, \theta)}$ obtained for all globally equivalent sets of states and for all final transformations $(\sigma, \delta, \theta)$ and let $N_{F}$ be the number of finitary states of $B$. We define $\Lambda$ as the integer $\operatorname{Sup}\left(\lambda, N_{F}\right)$.

As in the top-down case $[1,2,3]$, in order to eliminate these differences $\widehat{B}_{E_{(\sigma, \delta, \theta)}}$ $\widehat{B}_{F_{(\sigma, \delta, \theta)}}$ in the computations of some elements of $\widehat{B}_{E}$ (equal to $\widehat{B}_{F}$ ) we will consider as atomic any term an output tree of which is a ground term of height less than or equal to $A$. We do not compute this bound $A$ but, even if the construction is defined for any natural number $\nu$, the correctness of the results is connected to a large enough value of this integer.

\section{$3.2 \nu$-Semi-normalized Forms}

For any natural number $\nu$, we associate with any transducer $B$ its $\nu$-seminormalized form, denoted by $B^{\nu}$, and for which any couple of trees (or subtrees) $(t, u)$ with $\pi(t), \pi(u) \leq \nu$ is considered as atomic, that is to say is considered as a couple of new letters.

Let $B=<\Sigma, \Delta, Q, F, R>, B^{\nu}=<\Sigma^{\nu}, \Delta^{\nu}, Q^{\nu}, F^{\nu}, R^{\nu}>$ is constructed as follows:

- $Q^{\nu}=\left\{q^{<\nu}\right.$ and $\left.q^{\nu} \mid q \in Q\right\}$ and $F^{\nu}=\left\{q^{<\nu}\right.$ and $\left.q^{\nu} \mid q \in F\right\}$.

- Rules of $R^{\nu}$ which simulate computations producing trees of height $\leq \nu$ :

${ }^{1}$ and there is no computation $t_{0}\left(q_{1}\left(x_{1}\right), . ., q_{n}\left(x_{n}\right)\right) \stackrel{*}{\longmapsto_{B}} t^{\prime}\left(q_{1}^{\prime}\left(t_{1}^{\prime}\right), . ., q_{m}^{\prime}\left(t_{m}^{\prime}\right)\right)$ where $t^{\prime}$ is a prefix of $t_{0}$ and such that $t^{\prime}\left(q_{1}^{\prime}\left(x_{1}\right), . ., q_{m}^{\prime}\left(x_{m}\right)\right) \stackrel{*}{\longmapsto} B q(u)$ 


\begin{tabular}{|c|c|}
\hline Computations in $B$ & New letters and associated rules of $B^{\nu}$ \\
\hline $\begin{array}{c}t \stackrel{*}{\longmapsto} B q(u) \\
\pi(t)=\pi(u)<\nu\end{array}$ & $\begin{array}{l}t \rightarrow q^{<\nu}(u) \quad \in R^{\nu} \\
t \in \Sigma^{\nu} \text { and } u \in \Delta^{\nu}\end{array}$ \\
\hline $\begin{array}{c}t \stackrel{*}{\longmapsto} B q(u) \\
\pi(t)=\pi(u)=\nu\end{array}$ & $\begin{array}{c}t \rightarrow q^{\nu}(u) \quad \in R^{\nu} \\
t \in \Sigma^{\nu} \text { and } u \in \Delta^{\nu}\end{array}$ \\
\hline $\begin{array}{c}t_{0}\left(q_{1}\left(x_{1}\right), . ., q_{n}\left(x_{n}\right)\right) \stackrel{{ }^{*}}{\longmapsto} B q(u) \\
\pi\left(t_{0}\left(x_{1}, . ., x_{n}\right)\right)=\pi(u)<\nu^{1}\end{array}$ & $\begin{array}{c}t_{0}\left(q_{1}^{\diamond}\left(x_{1}\right), \ldots, q_{n}^{\diamond}\left(x_{n}\right)\right) \rightarrow q^{<\nu}(u) \in R^{\nu} \\
q_{i}^{\diamond} \text { is either } q_{i}^{<\nu} \text { or } q_{i}^{\nu}, t_{0} \in \Sigma_{n}^{\nu} \text { and } u \in \Delta_{0}^{\nu}\end{array}$ \\
\hline $\begin{array}{l}t_{0}\left(q_{1}\left(x_{1}\right), \ldots, q_{n}\left(x_{n}\right)\right) \stackrel{*}{{ }^{*}} B q(u) \\
\pi\left(t_{0}\left(x_{1}, . ., x_{n}\right)\right)=\pi(u)=\nu^{1}\end{array}$ & $\begin{array}{c}t_{0}\left(q_{1}^{\diamond}\left(x_{1}\right), \ldots, q_{n}^{\diamond}\left(x_{n}\right)\right) \rightarrow q^{\nu}(u) \in R^{\nu} \\
q_{i}^{\diamond} \text { is either } q_{i}^{<\nu} \text { or } q_{i}^{\nu}, t_{0} \in \Sigma_{n}^{\nu} \text { and } u \in \Delta_{0}^{\nu}\end{array}$ \\
\hline
\end{tabular}

- In order to control the depth of the trees which are transformed,

for any rule $\sigma\left(q_{1}\left(x_{1}\right), . ., q_{n}\left(x_{n}\right)\right) \rightarrow q\left(\delta\left(x_{\theta(1)}, . ., x_{\theta(m)}\right)\right)$ in $R$ we will have in $R^{\nu}$ the rules $\sigma\left(q_{1}^{\diamond}\left(x_{1}\right), \ldots, q_{n}^{\diamond}\left(x_{n}\right)\right) \rightarrow q^{\nu}\left(\delta\left(x_{\theta(1)}, . ., x_{\theta(m)}\right)\right)$ where

- $\sigma \in \Sigma_{n}^{\nu}$ and $\delta \in \Delta_{m}^{\nu}$

- there exists at least $j \in[n]$ such that $q_{j}^{\diamond}$ is of the form $q_{j}^{\nu}$ and for any $i \in[n]$ with $i \neq j, q_{i}^{\diamond}$ is either $q_{i}^{<\nu}$ or $q_{i}^{\nu}$.

So, these non-ground rules only apply for output terms of height greater than $\nu$.

Example 2 (continued) For $\nu=1$, the $\nu$-semi-normalized form of $B_{1}$ (page 5 ) is defined by:

Rules producing ground terms:

$$
\begin{aligned}
& \bar{a} \rightarrow q_{1}^{<1}(e) \quad \bar{a} \rightarrow q_{2}^{<1}(e) \\
& a\left(q_{1}^{\prime \prime}<1(x)\right) \rightarrow q_{1}^{<1}(e) \quad a\left(q_{1}^{\prime \prime 1}(x)\right) \rightarrow q_{1}^{<1}(e) \\
& \bar{a} \rightarrow q_{1}^{\prime}<1(\bar{\alpha}) \quad \bar{a} \rightarrow q_{2}^{\prime<1}(\bar{\beta}) \\
& a\left(q_{2}^{\prime<1}(x)\right) \rightarrow q_{2}^{<1}(e) \\
& a\left(q_{2}^{\prime 1}(x)\right) \rightarrow q_{2}^{<1}(e) \\
& a(\bar{a}) \rightarrow q_{1}^{\prime \prime}(\alpha(\bar{\alpha})) \quad \sigma(., \bar{a}) q_{1}^{<1}(x) \rightarrow q^{1}(\delta(e, e)) \\
& a(\bar{a}) \rightarrow q_{2}^{\prime 1}(\beta(\beta)) \quad \sigma(\bar{a}, .) q_{2}^{<1}(x) \rightarrow q^{1}(\delta(e, e)) \\
& \left.\sigma(\bar{a}, \vec{a})] \rightarrow q^{1}(\delta(e, e)) \quad \sigma\left(q_{1}^{<1}(x), q_{2}^{<1}(y)\right) \rightarrow q^{1}(\delta(e, e)]\right) \\
& \text { Other rules: }\left\{\begin{array}{l}
a\left(q_{1}^{\prime 1}(x)\right) \rightarrow q_{1}^{\prime \prime} 1(\alpha(x)) \quad a\left(q_{1}^{\prime \prime}(x)\right) \rightarrow q_{1}^{\prime 1}(\alpha(x)) \\
a\left(q_{2}^{\prime 1}(x)\right) \rightarrow q_{2}^{\prime 1}(\beta(x))
\end{array}\right.
\end{aligned}
$$

Trees written into boxes are now considered as new letters. Note that we just mention the usable rules $\square$.

Lemma 2. Let $B$ be a letter-to-letter bottom-up transducer. There exists a natural number $\Lambda$ such that, for any couple $(E, F)^{1}$ of globally equivalent sets of states, for any final transformation $(\sigma, \delta, \theta), \widehat{B}_{E_{(\sigma, \delta, \theta)}^{A}}^{A}=\widehat{B}_{F_{(\sigma, \delta, \theta)}}^{A}$.

PRoof: Immediate from corollary 1 and the previous construction.

${ }^{1}$ To avoid a multiplication of notations, for any set $E$ of states of $B$, the set of states $\left\{q^{\nu}, q^{<\nu} \mid q \in E\right\}$ of $B^{\nu}$ is also denoted by $E$. 
We now prove that, when $\nu$ is large enough, for equivalent states of this $\nu$-seminormalized form, the same torsions can be applied (except eventually for subtrees which are next deleted). In order to formalize this fact, from the transducer $B^{\nu}$ we introduce a new form, denoted by $B^{\nu, d}$, for which the torsions applied in a computation are encoded in the nodes of the output tree (the nodes of the output trees are "decorated" by these torsions).

\section{Construction of $B^{\nu, d}$}

From $B^{\nu}=<\Sigma^{\nu}, \Delta^{\nu}, Q^{\nu}, F^{\nu}, R^{\nu}>$, we construct $B^{\nu, d}=<\Sigma^{\nu}, \Delta^{\nu, d}, Q^{\nu}, F^{\nu}$, $R^{\nu, d}>$ where $\Delta^{\nu, d}$ and $R^{\nu, d}$ are defined by:

$$
\begin{aligned}
& \sigma\left(q_{1}\left(x_{1}\right), \ldots, q_{n}\left(x_{n}\right)\right) \rightarrow q\left(\delta_{\theta}\left(x_{\theta(1)}, \ldots, x_{\theta(m)}\right)\right) \in R^{\nu, d} \text { and } \delta_{\theta} \in \Delta^{\nu, d} \\
& \text { if } \sigma\left(q_{1}\left(x_{1}\right), \ldots, q_{n}\left(x_{n}\right)\right) \rightarrow q\left(\delta\left(x_{\theta(1)}, . ., x_{\theta(m)}\right)\right) \in R^{\nu}, \text { and any ground rule } \\
& \sigma\left(q_{1}\left(x_{1}\right), \ldots, q_{n}\left(x_{n}\right)\right) \rightarrow q^{\diamond}(\delta) \text { of } R^{\nu} \text { is a rule of } R^{\nu, d} .
\end{aligned}
$$

In the following, for any couple $(t, u)$ of $\widehat{B}^{\nu}$, we will denote by $\left(t, u^{d}\right)$ the corresponding couple of $\widehat{B}^{\nu, d}$.

To establish the fact that, when $\nu$ is large enough, we have $\widehat{B}_{E}=\widehat{B}_{F} \Leftrightarrow \widehat{B}_{E}^{\nu, d}=$ $\hat{B}_{F}^{\nu, d}$ we will use the following technical lemma:

Lemma 3. Let $E$ and $F$ be two sets of globally equivalent states of the $\nu$-seminormalized form $B^{\nu}$ of a transducer $B$. When $\nu$ is large enough, for any couple $(t, u)$ of $\widehat{B}_{E}^{\nu}\left(=\widehat{B}_{F}^{\nu}\right)$, for any prefix $u_{0}$ of $u$, for any state $q$ of $E$, for any computation

$$
t_{0}\left(t_{1}, . ., t_{n}\right) \stackrel{*}{\longmapsto} B^{\nu} t_{0}\left(q_{1}\left(u_{1}\right), . ., q_{n}\left(u_{n}\right)\right) \stackrel{*}{\longmapsto} B^{\nu, d} q\left(u_{0}^{d}\left(u_{\theta(1)}, . ., u_{\theta(m)}\right)\right)
$$

there exists $k \in F$ such that

$$
t_{0}\left(t_{1}, . ., t_{n}\right) \stackrel{*}{\longmapsto} B^{\nu} t_{0}\left(k_{1}\left(u_{1}\right), . ., k_{n}\left(u_{n}\right)\right) \stackrel{*}{\longmapsto} B^{\nu, d} k\left(u_{0}^{d}\left(u_{\theta(1)}, . ., u_{\theta(m)}\right)\right) .
$$

Proof: By induction on the prefix $u_{0}$ of $u$. Let $\nu=\Lambda$ as defined in corollary 1 .

- From the construction of $B^{A}$ and by means of lemma 2 the property is true when the prefix of $u$ is reduced to a letter of $\Delta^{A}$.

- Assume now that property is true for a prefix $u_{0}$ of $u$, that is to say for couples of the form $\left(t, u_{0}\left(u_{1}, . ., u_{m}\right)\right)$ of $\widehat{B}_{E}^{A}$ and let us show that it is true again for a larger prefix.

For any $j \in[m]$ we consider the sets

$$
\begin{aligned}
& Q_{j}=\left\{q_{r} \mid t_{0}\left(q_{1}\left(x_{1}\right), . ., q_{r}\left(x_{r}\right), \ldots, q_{n}\left(x_{n}\right)\right) \stackrel{*}{\longrightarrow} B^{\Lambda, d} q\left(u_{0}^{d}\left(x_{\theta(1)}, . ., x_{r}, . ., x_{\theta(m)}\right)\right)\right. \text {, } \\
& \text { with } q \in E \text { and } r=\theta(j)\} \text { and } \\
& K_{j}=\left\{k_{r} \mid t_{0}\left(k_{1}\left(x_{1}\right), . ., k_{r}\left(x_{r}\right), \ldots, k_{n}\left(x_{n}\right)\right) \stackrel{*}{\longmapsto} B^{\Lambda, d} k\left(u_{0}^{d}\left(x_{\theta(1)}, . ., x_{r}, . ., x_{\theta(m)}\right)\right)\right. \text {, } \\
& \text { with } k \in F \text { and } r=\theta(j)\} \text {. }
\end{aligned}
$$

First, let us show that for any $j \in[\mathrm{m}], Q_{j}$ and $K_{j}$ are globally equivalent sets of states. Assume that there exists $j \in[m]$ such that $Q_{j}$ and $K_{j}$ are not globally equivalent. There would exist at least one couple $(\bar{\tau}, \bar{\mu})$ in $\widehat{B}_{Q_{3}}-\widehat{B}_{K_{3}}$ such that $t_{0}\left(\bar{t}_{1}, . ., \bar{\tau}, . ., \bar{t}_{n}\right) \stackrel{*}{\longmapsto}_{B^{\Lambda}} t_{0}\left(q_{1}\left(\bar{u}_{1}\right), . ., q_{\theta(j)}(\bar{\mu}), . ., q_{n}\left(\bar{u}_{n}\right)\right) \stackrel{*}{*}_{B^{\Lambda, d}} q\left(u_{0}^{d}\left(\bar{u}_{\theta(1)}, . ., \bar{\mu}, .\right.\right.$. $\left.\left.6 \bar{u}_{\theta(m)}\right)\right)$ ) when, because $(\bar{\tau}, \bar{\mu}) \notin \hat{B}_{K}$, there does not exist any computation from 
$t_{0}\left(\bar{t}_{1}, . ., \bar{\tau}, . ., \bar{t}_{n}\right)$ which leads to $\left.k\left(u_{0}^{d}\left(\bar{u}_{\theta(1)}, . ., \bar{\mu}, . ., \bar{u}_{\theta(m)}\right)\right)\right)$.

It would contradict the assumption that property holds for $u_{0}$ and so for any $j \in[m]$ the sets of states $Q_{j}$ and $K_{j}$ are globally equivalent.

Thus, for any $j \in[m]$, for any state $q^{\prime} \in Q_{j}$, there exists at least one state $k^{\prime} \in K_{j}$ such that the computations $t_{\theta(j)} \stackrel{*}{\longmapsto} q^{\prime}\left(u_{\theta(j)}\right)$ and $t_{\theta(j)} \stackrel{*}{\longmapsto} k^{\prime}\left(u_{\theta(j)}\right)$ are realized with the same final torsions (first point of this proof). Consequently the property is also satisfied for a larger prefix $\left(u_{0}\left(x_{1}, \ldots, v_{j}, \ldots, x_{m}\right)\right.$ with $v_{j}$ root of $u_{j}$ ).

Proposition 1. Let $B$ be a bottom-up transducer. When $\nu$ is large enough, for any couple $(E, F)$ of sets of states, $\widehat{B}_{E}^{\nu, d}=\widehat{B}_{F}^{\nu, d} \Leftrightarrow \widehat{B}_{E}=\widehat{B}_{F}$.

Hint of Proof: We have obviously $\widehat{B}_{E}^{\nu, d}=\widehat{B}_{F}^{\nu, d} \Rightarrow \widehat{B}_{E}=\widehat{B}_{F}$. Conversely, we use the previous lemma (using as prefix of a tree the tree itself).

\section{$3.3 \nu$-normalized forms}

In the previous section proposition 1 established the fact that, when $\nu$ is large enough, for any transducer $B$, for globally equivalent sets of states $E$ and $F$, all computations can be realized with the same torsions in the $\Lambda$-semi-normalized form $B^{\nu, d}$. So if some subtrees are deleted or duplicated in a computation of a couple $(t, u)$ of $B_{E}^{\nu, d}$, we claim that there exists a computation of $(t, u)$ in $B_{F}^{\nu, d}$ which delete or duplicate the same subtrees. Because torsions have been encoded in the nodes of the output trees, it is not significant to apply them effectively. These facts lead us to define a $\nu$-normalized form $B^{\nu, n}$ which is a torsion-free transducer.

For the sake of comprehension, we describe the construction by means of examples where only one kind of torsion occurs:

- Permutation: from any rule of the form $\sigma\left(q_{1}\left(x_{1}\right), q_{2}\left(x_{2}\right)\right) \rightarrow q\left(\delta_{\theta}\left(x_{2}, x_{1}\right)\right)$, we construct the rule $\sigma\left(q_{1}\left(x_{1}\right), q_{2}\left(x_{2}\right)\right) \rightarrow q\left(\delta_{\theta}\left(x_{1}, x_{2}\right)\right)$.

- Non-linearity: by using a non-linear rule, a bottom-up transducer can only generate identical copies and so we will only keep one of them. So, from the rule $\sigma\left(q_{1}(x)\right) \rightarrow q\left(\delta_{\theta}(x, x)\right)$ we construct $\sigma\left(q_{1}(x)\right) \rightarrow q\left(\delta_{\theta}^{\prime}(x)\right)$.

- Deletion: A bottom-up transducer has the ability of inspecting a subtree before deleting it. So, for instance, to any rule of the form $\sigma\left(q_{1}\left(x_{1}\right), q_{2}\left(x_{2}\right)\right) \rightarrow$ $q\left(\delta_{\theta}\left(x_{1}\right)\right)$ we substitute the non-deleting rule $\sigma\left(q_{1}\left(x_{1}\right), \bar{q}_{2}\left(x_{2}\right)\right) \rightarrow q\left(\delta_{\theta}^{\prime}\left(x_{1}, x_{2}\right)\right)$ where the transformation associated with this new state $\bar{q}_{2}$ is the identity that is to say the set $\left\{(t, t) \mid t \in \operatorname{dom}\left(\widehat{B}_{q 2}^{\nu, d}\right)\right\}$. Note that, in particular, we have $\operatorname{dom}\left(\hat{B}_{q_{2}}^{\nu, d}\right)=\operatorname{dom}\left(\hat{B}_{\bar{q}_{2}}^{\nu, d}\right)$.

Moreover, for any state $q$, in order to realize the identity transformation on $\operatorname{dom}\left(\widehat{B}_{q}\right)$, from any rule $\sigma\left(q_{1}\left(x_{1}\right), . ., q_{n}\left(x_{n}\right)\right) \rightarrow q\left(\delta_{\theta}\left(x_{\theta(1)}, \ldots, x_{\theta(m)}\right)\right)$ of $B^{\nu, d}$, we construct the rule $\sigma\left(\bar{q}_{1}\left(x_{1}\right), . ., \bar{q}_{n}\left(x_{n}\right)\right) \rightarrow \bar{q}\left(\sigma\left(x_{1}, \ldots, x_{n}\right)\right)$. 
As these phenomena can be mixed in a same rule, the construction in the general case is a combinaison of the previous ones.

Example 2 (continued) We only give here the usable rules of the 1-normalized form of $B_{1}$. States have been renamed.

$$
\begin{aligned}
& \left.\left.\sigma(., \bar{a})] r_{1}(x) \rightarrow q(\delta(e, e)]_{1}(x)\right) \quad \sigma(\bar{a}, .) r_{2}(x) \rightarrow q(\delta(e, e)]_{1}(x)\right) \\
& \left.\sigma(\bar{a}, \bar{a}) \rightarrow q(\delta(e, e)) \quad \sigma\left(r_{1}(x), r_{2}(y)\right) \rightarrow q(\delta(e, e)]_{2}(x, y)\right) \\
& \bar{a} \rightarrow r_{1}(\bar{a}) \quad a(\bar{a}) \rightarrow r_{3}(\underline{a(\bar{a})}) \quad a\left(r_{3}(x)\right) \rightarrow r_{1}(a(x)) \\
& a\left(r_{3}(x)\right) \rightarrow r_{5}(a(x)) \quad a\left(r_{5}(x)\right) \rightarrow r_{3}(a(x)) \\
& \left.\left.\bar{a} \rightarrow r_{2}(\bar{a}) \quad a(\bar{a})\right] \rightarrow r_{2}([a(\bar{a})]) \quad a(\bar{a}) \rightarrow r_{4}(a(\bar{a})]\right) \\
& a\left(r_{4}(x)\right) \rightarrow r_{4}(a(x)) \quad a\left(r_{4}(x)\right) \rightarrow r_{2}(a(x))
\end{aligned}
$$

Note that $\delta(e, e)]_{1}$ is a new letter of rank 1 and $\left.\delta(e, e)\right]_{2}$ is of rank 2. Moreover, due to the deleting rules of $B_{1}$, many rules of this normalized form are automaton rules like.

By means of proposition 1 we know that, when $\nu$ is large enough, for any couple $(E, F)$ of sets of states, $\widehat{B}_{E}^{\nu, d}=\widehat{B}_{F}^{\nu, d} \Leftrightarrow \widehat{B}_{E}=\widehat{B}_{F}$. Now, by the previous construction, equivalence of states is obviously preserved and so we obtain:

Proposition 2. Let $B$ be a letter-to-letter bottom-up transducer. When $\nu$ is large enough, for any couple of sets of states $(E, F), \widehat{B}_{E}=\widehat{B}_{F} \Leftrightarrow \widehat{B}_{E}^{\nu, n}=\widehat{B}_{F}^{\nu, n}$.

\subsection{Decidability of equivalence}

Using the previous result, we can now prove:

Theorem 1. Equivalence of letter-to-letter bottom-up transducers is decidable.

Proof: Let $B=<\Sigma, \Delta, Q, F, R>$ and $B^{\prime}=<\Sigma, \Delta, Q^{\prime}, F^{\prime}, R^{\prime}>$ with $Q \cap Q^{\prime} \neq$ $\emptyset$ (otherwise, we can relabel them). As equivalence is decidable for bottom-up relabelings [4], from proposition 2 applied to the sets of states $F$ and $F^{\prime}$ (of $B \cup B^{\prime}$ ) we obtain that equivalence of $B$ and $B^{\prime}$ is semi-decidable. Because non-equivalence is obviously semi-decidable, we conclude.

\section{Compositions of letter-to-letter tree transducers}

Following the Engelfriet's works [7], we consider the tree transformations realized by compositions of two letter-to-letter tree transducers: a first phase accomplished by a bottom-up transducer followed by a second one realized by a top-down transducer. These compositions can, for instance perform some optimizations on relational databases as it is illustrated in the following example. 
Example 3 We assume the reader is familiar with the relational data model. We consider here the set-of-mappings viewpoint in which columns of all relations have attributes names and tuples are regarded as mapping from attributes to values. Let us consider a relational scheme $\mathcal{R}$ constituted of the relations $R_{1}, R_{2}, \ldots, R_{n}$. Let be $\mathcal{A}=\left\{a_{1}, a_{2}, \ldots, a_{p}\right\}$ the set of the attributes of these relations. We only consider here the following operators of the relational algebra:

Selection: Select $F$ where $\mathrm{F}$ is a formula involving attributes referred by their name. Let us denote by $\nu(F)$ the set of attributes that appear in formula $F$.

Natural join: $\bowtie$. $R \bowtie S$ denotes the join of $R$ and $S$ on each attribute that is both in $R$ and $S$.

Projection: the projection of relation $R$ on $x$ will be denoted by $R[x]$.

"Selection" and "Join" can be composed sequentially with a projection on a subset $x$ of attributes. We will use the shorthands Select $F[x], \bowtie[x]$ for these compositions.

With any relational expression, we can associate its parse tree, so query optimization by algebraic manipulation can be seen as tree transformation. We show that the move of the projections as far down the tree as possible can be obtained by such a composition of transducers.

\section{Letter to letter bottom-up transducer}

This first transformation labels the natural join operators with the couple of sets of attributes of the joined relations. The rules of this transducer correspond also to validity checks.

Let $T_{\uparrow}=<Q, \Sigma, \Delta, F, \mathcal{R}>$ where

- the states of $Q$ are the subsets of $\mathcal{A} ; F=Q$

$-\Sigma_{0}=\left\{R_{1}, R_{\imath}[x] / i \in[n], x \subset \mathcal{A}\right\}$;

$\Sigma_{1}=\left\{\right.$ Select $_{x}$, Select $\left.x[y] / x, y \subset \mathcal{A}\right\} ;$ the label Select $x$ is associated with any operator Select $F$ where $\nu(F)=x ; \quad \Sigma_{2}=\{\bowtie\} \cup\{\bowtie[x] / x \subset \mathcal{A}\}$

- $\Delta_{0}=\left\{R_{\imath} / i \in[u]\right\} ; \Delta_{1}=\{$ Select $x / x \subset \mathcal{A}\} ; \Delta_{2}=\left\{\aleph_{(y, z)} / y, z \subset \mathcal{A}\right\}$

- rules of $\mathcal{R}$ are constructed as follows:

- Let $s$ be the set of attributes of $R . R \rightarrow s(R), R[x] \rightarrow x(R)$ iff $x \subset s$

- Select $x(s) \rightarrow s\left(\right.$ Select $\left._{x}\right)$ iff $x \subset s$, Select $_{x}[r](s) \rightarrow r\left(\right.$ Select $\left._{x}\right)$ iff $(x \cup r) \subset s$

- $\left(s, s^{\prime}\right) \rightarrow s^{\prime \prime}\left(\aleph_{\left(s, s^{\prime}\right)}\right)$ with $s^{\prime \prime}=s \cup s^{\prime}, \bowtie[r]\left(s, s^{\prime}\right) \rightarrow r\left(\aleph_{\left(s, s^{\prime}\right)}\right)$ iff $r \subset\left(s \cup s^{\prime}\right)$

Letter to letter top-down transducer

This transducer associates with each operator which occurs in the expression, the projection onto the minimal set of attributes that is useful to evaluate the expression. Its input alphabet is $\Delta$ and its output alphabet $\Sigma$. The initial state of a top-down computation will be the state reached at the end of the bottom-np computation. So its rules have the following forms:

$s(R) \rightarrow R[s], s($ Select $x) \rightarrow$ Select $_{x}[s](x \cup s)$,

$s\left(\aleph_{\left(r_{1}, r_{2}\right)}\right) \rightarrow \bowtie[s]\left(r_{1} \cap\left(r_{2} \cup s\right), r_{2} \cap\left(r_{1} \cup s\right)\right)$ for $s \subset \mathcal{A}$ and $s \neq \emptyset$

\section{Application}

Let us consider the relational database scheme:

Customer( $C \#, C_{-}$name, $C_{-}$adress), Order(O\#,C\#,O_date), Include(O\#, $A \#$, quantity), Article(A\#, A_description, A_price, S\#), Supplier(S\#, S_name, S-adress). C\#, O\#, A\#, S\# refer primary keys on relations Customer, Order, Article, Supplier.

Let us now consider the query: Which customers have given orders for articles supplied by a given supplier?

This query is turned into a relational expression. The following figure presents the parse 
tree of this expression versus its optimized tree obtained by the considered two-way transducer.

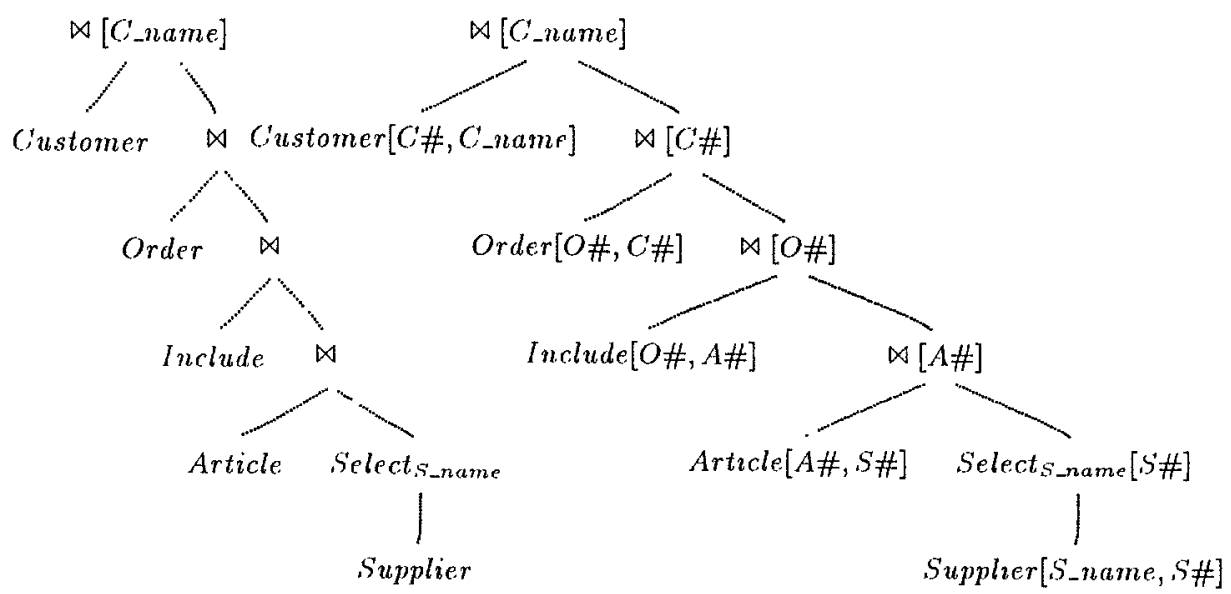

We show that compositions correspond closely to transformations realized by letter-to-letter top-down tree transducers with regular look-ahead. In [7], J. Engelfriet proved that $T^{R}-F S T \subseteq D B-L A B \circ T-F S T$. Here, we have

Lemma 4. $T^{R}-L L \subseteq B-L L \circ T-L L$.

PRoof: By applying the construction described in the proof of theorem 2.6 of [7] to the letter-to-letter case, we immediately obtain that $T^{R}-L L \subseteq D B$ $L A B \circ T-L L$. Now $D B-L A B \subseteq B-L L$ and so $T^{R}-L L \subseteq B-L L \circ T-L L$.

In order to obtain the reverse property ( $\left.B-L L \circ T-L L \subseteq T^{R}-L L\right)$, with any couple $(B, T)$ of letter-to-letter transducers (where $B$ is a bottom-up transducer and $T$ is a top-down one), we associate the letter-to-letter top-down tree transducer with regular look-ahead $T^{R}$ the rules of which are constructed as follows:

$(q, k)\left(\sigma\left(x_{1}, . ., x_{n}\right)\right) \rightarrow \gamma\left(\left(q_{\theta(\mu(1))}, k_{1}\right)\left(x_{\theta(\mu(1))}\right), . .,\left(q_{\theta(\mu(p))}, k_{p}\right)\left(x_{\theta(\mu(p))}\right)\right),<L_{1}, . .$, $L_{n}>\in T^{R}$ if and only if

$\sigma\left(q_{1}\left(x_{1}\right), . ., q_{n}\left(x_{n}\right)\right) \rightarrow q\left(\delta\left(x_{\theta(1)}, . ., x_{\theta(m)}\right)\right)$ is a rule of $B$, with for any $i \in[n]$, $\operatorname{dom}\left(\widehat{B}_{q_{v}}\right)=L_{i}$ and $k\left(\delta\left(x_{1}, . ., x_{m}\right)\right) \rightarrow \gamma\left(k_{1}\left(x_{\mu(1)}\right), . ., k_{p}\left(x_{\mu(p)}\right)\right)$ is a rule of $T$.

The so constructed transducer with look-ahead verifies the following property:

Lemma 5. For any tree $t$, for any prefix $t_{0}$ of $t$,

$$
\begin{aligned}
& t_{0}\left(t_{1}, \ldots, t_{n}\right) \stackrel{*}{\longmapsto} B t_{0}\left(q_{1}\left(u_{1}\right), \ldots, q_{n}\left(u_{n}\right)\right) \stackrel{{ }^{*}}{\longrightarrow} B q\left(u_{0}\left(u_{\theta(1)}, . ., u_{\theta(m)}\right)\right) \\
& \text { and } k\left(u_{0}\left(u_{\theta(1)}, . ., u_{\theta(m)}\right)\right) \stackrel{*}{\longrightarrow} T v_{0}\left(k_{1}\left(u_{\theta(\mu(1))}\right), . ., k_{p}\left(u_{\theta(\mu(p))}\right)\right)
\end{aligned}
$$

if and only if

$$
\begin{aligned}
& (q, k)\left(t_{0}\left(t_{1}, . ., t_{n}\right)\right) \stackrel{*}{\longrightarrow} T_{R} v_{0}\left(\left(q_{\theta(\mu(1))}, k_{1}\right)\left(t_{\theta(\mu(1))}\right), \ldots,\left(q_{\theta(\mu(p))}, k_{p}\right)\left(t_{\theta(\mu(p))}\right)\right) \\
& \text { with, for any } i \in[n], t_{i} \in \operatorname{dom}\left(\widehat{B}_{q_{z}}\right) .
\end{aligned}
$$


The proof, by induction on $t$, is given in the appendix.

Theorem 2. $T^{R}-L L=B-L L \circ T-L L$.

Proof: By means of lemma 5 with as a prefix of any tree $t$, the tree $t$ itself, we have $B-L L \circ T-L L \subseteq T^{R}-L L$. Then using lemma 4, we conclude.

Remark If we consider the equivalence problem for letter-to-letter top-down transducers with regular look-ahead, our method does not allow to investigate the general (deleting and non-linear) case (because it fails in the general letterto-letter top-down case [3]). But we have: Equivalence of letter-to-letter top-down transducers with regular look-ahead is decidable in the linear case and in the nondeleting one.

In fact, J. Engelfriet established the equality $L T^{R}-F S T=L B-F S T$ (theorem 2.8 in [7]). This equality obviously holds in the letter-to-letter case. Now equivalence is decidable in $L B-L L$ (theorem 1) then it is in $L T^{R}-L L$. On the other hand, we have obviously $N d T^{R}-L L=N d T-L L$. Equivalence being decidable in $N d T-L L$ [3], we conclude.

\section{Extensions}

Non-linearity is an important phenomenon which often appears in trees, for instance in the rewrite rules $-(x, x) \rightarrow 0$ and $*(x, x) \rightarrow s q r(x)$. So a natural extension of our work would be to investigate letter-to-letter bottom-up transducers the rules of which can have non-linear left-hand sides. In this section, we only consider a restricted case.

\subsection{Definitions}

A generalized letter-to letter bottom-up tree transducer is a 5-tuple $B=<\Sigma, \Delta, Q$, $F, R>$ where $\Sigma, \Delta, Q$ and $F$ are defined as for "classical" bottom-up tree transducer and $R$ is a finite set of rules of the form $\sigma\left(q_{1}\left(x_{\theta(1)}\right), \ldots, q_{n}\left(x_{\theta(n)}\right)\right) \mapsto$ $q\left(\delta\left(x_{\mu(1)}, . ., x_{\mu(m)}\right)\right)$ where $\theta$ is a surjective mapping from $[n]$ to $[p]$ (with $p \leq n$ ) and $\mu$ is a mapping from $[m]$ to $[p]$. By gB-LL, we denote the class of all generalized Letter-to Letter Bottom-up tree transducer.

In the following, we restrict ourselves to generalized letter-to letter, called onestate transducers, for which $Q$ is reduced to one state.

We first define an order relation on the set of rules of any transducer such that a rule $r$ is said to be "more general" than a rule $r^{\prime}$ if everywhere the rule $r^{\prime}$ is applied, the rule $r$ can be used with the same result.

Formally, let $r: \sigma\left(q\left(x_{\theta(1)}\right), . ., q\left(x_{\theta(n)}\right)\right) \rightarrow q\left(\delta\left(x_{\mu(1)}, . ., x_{\mu(m)}\right)\right)$ and $r^{\prime}: \sigma\left(q\left(x_{\theta^{\prime}(1)}\right), \ldots, q\left(x_{\theta^{\prime}(n)}\right)\right) \rightarrow q\left(\delta\left(x_{\mu^{\prime}(1)}, . ., x_{\mu^{\prime}(m)}\right)\right)$ be two rules of a one-state transducer. $r$ is more general than $r^{\prime}$ if there exists a mapping $\phi$ such that $\phi \circ \theta=\theta^{\prime}$ and $\phi \circ \mu=\mu^{\prime}$. We write $r \succeq r^{\prime}\left(\right.$ or $r^{\prime} \preceq r$ ). 
Example Let $r_{1}: \sigma\left(q\left(x_{1}\right), q\left(x_{2}\right), q\left(x_{1}\right), q\left(x_{3}\right)\right) \rightarrow q\left(\delta\left(x_{1}, x_{1}, x_{2}, x_{3}\right)\right)$, $r_{2}: \sigma\left(q\left(x_{1}\right), q\left(x_{2}\right), q\left(x_{1}\right), q\left(x_{2}\right)\right) \rightarrow q\left(\delta\left(x_{1}, x_{1}, x_{2}, x_{2}\right)\right), r_{3}: \sigma\left(q\left(x_{1}\right), q\left(x_{2}\right), q\left(x_{3}\right)\right.$, $\left.q\left(x_{1}\right)\right) \rightarrow q\left(\delta\left(x_{1}, x_{1}, x_{2}, x_{3}\right)\right)$; we have $r_{1} \succeq r_{2}$ but neither $r_{1} \succeq r_{3}$ nor $r_{3} \succeq r_{1}$.

A one-state generalized transducer is said to be cleaned if for any rule $r$ there does not exist any rule $r^{\prime}$ such that $r \succeq r^{\prime}$.

Property 2. With any one-state transducer $B$ of gB-LL we can associate $a$ cleaned one-state transducer $B^{\mathfrak{c}}$ which is equivalent to $B$.

PRoof: If $r$ and $r^{\prime}$ are two rules of a transducer $B$ such that $r \succeq r^{\prime}$ then anywhere $r^{\prime}$ is applied, we can apply $r$ without difference in the transformation and so $r^{\prime}$ is not useful. By suppressing such rules, we obtain a cleaned transducer which is equivalent to $B$.

\subsection{Equivalence of one-state transducers}

Theorem 3. Two one-state transducers of gB-LL are equivalent if and only if their cleaned forms are equal, and so their equivalence is decidable.

Proof: Let $B_{1}$ and $B_{2}$ be two equivalent one-state transducers and let $B_{1}^{c}$ and $B_{2}^{c}$ be their cleaned forms. To establish the equality $B_{1}^{c}=B_{2}^{c}$, we show that, for any rule $r: \sigma\left(q_{1}\left(x_{\theta(1)}\right), . ., q_{1}\left(x_{\theta(n)}\right)\right) \mapsto q_{1}\left(\delta\left(x_{\mu(1)}, . ., x_{\mu(m)}\right)\right)$ of $B_{1}^{c}$, with $\theta$ and $\mu$ mapping into $[p]$, there exists the same rule $\sigma\left(q_{2}\left(x_{\theta(1)}\right), . ., q_{2}\left(x_{\theta(n)}\right)\right)$ $\mapsto q_{2}\left(\delta\left(x_{\mu(1)}, . ., x_{\mu(m)}\right)\right)$ in $B_{2}^{c}$.

Let us consider the couples of trees $\left(t_{1}, u_{1}\right), \ldots,\left(t_{p}, u_{p}\right)$ with, for any $i$ and $j$ in $[p], \pi\left(u_{i}\right) \neq \pi\left(u_{j}\right)$ (it is possible if there exists at least a rule producing a letter of rank greater than $\left.0^{1}\right)$ and such that $\sigma\left(t_{\theta(1)}, . ., t_{\theta(n)}\right) \stackrel{{ }^{*}}{\longmapsto} B_{1}^{c}$ $\sigma\left(q_{1}\left(u_{\theta(1)}\right), . ., q_{n}\left(u_{\theta(n)}\right)\right) \mapsto B_{1}^{c} q_{1}\left(\delta\left(u_{\mu(1)}, . ., u_{\mu(m)}\right)\right)$.

$B_{1}$ and $B_{2}$ being equivalent there would exist in $B_{2}^{c}$ a rule $r^{\prime}$ such that $r^{\prime} \succeq r$. Let $r^{\prime}: \sigma\left(q_{2}\left(x_{\theta^{\prime}(1)}\right), \ldots, q_{2}\left(x_{\theta^{\prime}(n)}\right)\right) \mapsto q_{2}\left(\delta\left(x_{\mu^{\prime}(1)}, \ldots, x_{\mu^{\prime}(m)}\right)\right)$ ( $\theta^{\prime}$ mapping from $[n]$ to $[s])$ such that and let us consider the couples of trees $\left(t_{1}^{\prime}, u_{1}^{\prime}\right), . .,\left(t_{s}^{\prime}, u_{s}^{\prime}\right)$ with, for any $i$ and $j$ in $[s], \pi\left(u_{i}^{\prime}\right) \neq \pi\left(u_{j}^{\prime}\right)$ and such that $\sigma\left(t_{\theta^{\prime}(1)}, . ., t_{\theta^{\prime}(n)}\right) \stackrel{*}{\longmapsto} B_{2}^{c}$ $\sigma\left(q_{2}\left(u_{\theta^{\prime}(1)}\right), . ., q_{2}\left(u_{\theta^{\prime}(n)}\right)\right) \mapsto B_{2}^{c} q_{2}\left(\delta\left(u_{\mu^{\prime}(1)}, . ., u_{\mu^{\prime}(m)}\right)\right)$.

$B_{1}^{c}$ and $B_{2}^{c}$ being equivalent we would find in $B_{1}^{c}$ a rule $r^{\prime \prime}$ with $r^{\prime \prime} \succeq r^{\prime}$. Now $B_{1}^{c}$ is cleaned then $r=r^{\prime}=r^{\prime \prime}$.

Thus, if two one-state transducers are equivalent, their cleaned forms are equal. The converse is obviously true and so we conclude.

\section{References}

[1] Y. André and M. Danchet. Decidability of equivalence for a class of nondeterministic tree transducers. RAIRO, Theoretical Informatics and Applications. Vol 28, $\mathrm{n}^{\circ} 5,1994$, pp 447-463.

${ }^{1}$ the result is valid as soon as the output alphabet $\Delta$ contains two letters. On the contrary, it is not true if $\Delta$ is a singleton. That is the case, for instance, for the two transducers $\{\sigma(x, y) \rightarrow a, a \rightarrow a\}$ and $\{\sigma(x, x) \rightarrow a, a \rightarrow a\}$. In such situations, two transducers are equivalent if the same couples of letters appear in the rules. 
[2] Y. André and F. Bossut. Decidability of equivalence for linear letter-to-letter top-down tree transducers. Proceedings of FCT'93. LNCS 710, pp 142-151.

[3] Y. André and F. Bossut. On the equivalence problem for letter-to-letter topdown tree transducers. Submitted to publication.

[4] S. Bozapalidis. Alphabetic tree relations. T.C.S. 99, pp 177-211. 1992.

[5] J.L. Coquidé, M. Dauchet, R. Gilleron and S. Vágvölgyi. Bottom-up tree pushdown automata: classification and connection with rewrite systems. T.C.S. 127, pp 69-98. 1994.

[6] J. Engelfriet. Bottom-up and top-down tree transformations: a comparison. Mathematical system theory. Vol 9. pp 198-231. 1975.

[7] J. Engelfriet. Top-down Tree Transducers with Regular Look-ahead. Mathematical system theory. Vol 10. pp 289-303. 1977.

[8] J. Engelfriet and H. Vogler. Macro Tree Transducers. J.C.S.S. 31, pp 71-146. 1985.

[9] J. Engelfriet and H. Vogler. Modular Tree Transducers. T.C.S. 78, pp 267-303. 1991.

[10] Z. Esik. Decidability results concerning tree transducers I. Acta Cybernetica,Tom.5, Fasc.1, pp 1-20, Szeged, 1980.

[11] Z. Fülöp and S. Vágvölgyi. Variants of top-down tree transducers with lookahead. Mathematical Systems Theory 21. pp125-145. 1989.

[12] F. Gecseg and M. Steinby. Tree automata. Akademiai Kiado, Budapest. 1984.

[13] W.C. Rounds. Trees, transducers and transformations. Ph.D. Thesis. Standford University. 1968.

[14] H. Seidl. Equivalence of finite-valued bottom-up finite state tree transducers is decidable. Proceedings of CAAP'90. LNCS 431, pp 269-284.

[15] J.W. Thatcher. Generalized sequential machine maps. J.C.S.S. 4. 1970.

[16] Z. Zachar. The solvability of the equivalence problem for deterministic frontierto-root tree transducers. Acta Cybernetica, v.4, 1978, pp 167-177.

\section{Appendix}

Proof of lemma 5 It is by induction on the prefix of any tree $t$.

Induction base According to the construction, the property is true when $t_{0}$ is reduced to a letter.

Induction step Assume that property is true for a prefix $t_{0}$ of $t$ and let us show that it is true again for a larger prefix. Let $t=t_{0}\left(t_{1}, \ldots, t_{i}, . ., t_{n}\right)$ with $t_{i}=a\left(t_{1}^{\prime}, . ., t_{r}^{\prime}\right)$. So, we have:

$$
\begin{aligned}
& t_{0}\left(t_{1}, . ., a\left(t_{1}^{\prime}, \ldots, t_{r}^{\prime}\right), . ., t_{n}\right) \stackrel{*}{\mapsto_{B}} t_{0}\left(q_{1}\left(u_{1}\right), . ., a\left(q_{1}^{\prime}\left(u_{1}^{\prime}\right), . ., q_{r}^{\prime}\left(u_{r}^{\prime}\right)\right), . ., q_{n}\left(u_{n}\right)\right) \\
& \stackrel{*}{\longrightarrow} B t_{0}\left(q_{1}\left(u_{1}\right), \ldots, q_{i}\left(b\left(u_{\alpha(1)}^{\prime}, . ., u_{\alpha(s)}^{\prime}\right)\right), \ldots, q_{n}\left(u_{n}\right)\right) \\
& \mapsto_{B} \quad q\left(u_{0}\left(u_{\theta(1)}, . ., u_{\theta(m)}\right)\right)
\end{aligned}
$$

(so, for any $j \in[m]$ such that $\theta(j)=i u_{\theta(j)}=b\left(u_{\alpha(1)}^{\prime}, \ldots, u_{\alpha(s)}^{\prime}\right.$ ), for any $i \in[n]$

$t_{i} \in \operatorname{dom}\left(\widehat{B}_{q_{z}}\right)$, and for any $\left.i \in[r] t_{i}^{\prime} \in \operatorname{dom}\left(\widehat{B}_{q_{\imath}^{\prime}}\right)\right)$

and $k\left(u_{0}\left(u_{\theta(1)}, . ., u_{\theta(m)}\right)\right) \stackrel{*}{\longmapsto} T v_{0}\left(k_{1}\left(u_{\theta(\mu(1))}\right), . ., k_{p}\left(u_{\theta(\mu(p))}\right)\right)$. 
Let $\theta \circ \mu=\phi$ and assume that there exists at least $l$ such that $\phi(l)=i$. Thus $v_{0}\left(k_{1}\left(u_{\phi(1)}\right), . ., k_{l}\left(u_{\phi(l)}\right), . ., k_{p}\left(u_{\phi(p)}\right)\right) \mapsto_{B}$ $v_{0}\left(k_{1}\left(u_{\phi(1)}\right), \ldots, c\left(k_{1}^{\prime}\left(u_{\alpha(\beta(1))}^{\prime}\right), \ldots, k_{w}^{\prime}\left(u_{\alpha(\beta(w))}^{\prime}\right)\right), . ., k_{p}\left(u_{\phi(p)}\right)\right)$ $=v_{0}\left(k_{1}\left(u_{\phi(1)}\right), . ., c\left(k_{1}^{\prime}\left(u_{\gamma(1)}^{\prime}\right), . ., k_{w}^{\prime}\left(u_{\gamma(w)}^{\prime}\right)\right), . ., k_{p}\left(u_{\phi(p)}\right)\right)$ where $\gamma=\alpha \circ \beta$.

Note that if there does not exist any $l$ such that $\phi(l)=i$ for

i) there does not exist $i^{\prime}$ such that $\theta\left(i^{\prime}\right)=i$

ii) for any $i^{\prime}$ such that $\theta\left(i^{\prime}\right)=i$, there does not exist $i^{\prime \prime}$ such that $\mu\left(i^{\prime \prime}\right)=i$, we have a weaker form of the previous case. Let us point out that these cases justify the introduction of a regular look-ahead check.

These computations are equivalent to

i) by the induction hypothesis:

$\left(q_{b(l)}, k_{l}\right)\left(t_{\phi(l)}\right)=\left(q_{\phi(l)}, k_{1}\right)\left(a\left(t_{1}^{\prime}, \ldots, t_{r}^{\prime}\right)\right) \stackrel{*}{\longmapsto} c\left(\left(q_{\gamma(1)}^{\prime}, k_{1}^{\prime}\right)\left(u_{\gamma(1)}^{\prime}\right), \ldots,\left(q_{\gamma(w)}^{\prime}, k_{w}^{\prime}\right)\left(u_{\gamma(w)}^{\prime}\right)\right)$

ii) by the induction base:

$\left(q_{\phi(l)}, k_{l}\right)\left(t_{\phi(l)}\right)=\left(q_{\phi(l)}, k_{l}\right)\left(a\left(t_{1}^{\prime}, . ., t_{r}^{\prime}\right)\right) \stackrel{*}{\longmapsto} c\left(\left(q_{\gamma(1)}^{\prime}, k_{1}^{\prime}\right)\left(u_{\gamma(1)}^{\prime}\right), \ldots,\left(q_{\gamma(w)}^{\prime}, k_{w}^{\prime}\right)\left(u_{\gamma(w)}^{\prime}\right)\right)$

That is to say $(q, k)\left(t_{0}\left(t_{1}, . ., a\left(t_{1}^{\prime}, . ., t_{r}^{\prime}\right), . ., t_{n}\right)\right) \stackrel{*}{\longmapsto} T_{R}$ $v_{0}\left(\left(q_{\phi(1)}, k_{1}\right)\left(t_{\phi(1)}\right), . ., c\left(\left(q_{\gamma(1)}^{\prime}, k_{1}^{\prime}\right)\left(u_{\gamma(1)}^{\prime}\right), . .,\left(q_{\gamma(w)}^{\prime}, k_{w}^{\prime}\right)\left(u_{\gamma(w)}^{\prime}\right)\right), . .,\left(q_{\phi(p)}, k_{p}\right)\left(t_{\phi(p)}\right)\right)$ with for any $i \in[n] t_{2} \in \operatorname{dom}\left(\widehat{B}_{q_{2}}\right)$ and for any $i \in[r] t_{i}^{\prime} \in \operatorname{dom}\left(\hat{B}_{q_{2}^{\prime}}\right)$. So the property is true for the larger prefix $t_{0}\left(x_{1}, . ., a\left(x_{1}^{\prime}, \ldots, x_{r}^{\prime}\right), . ., x_{n}\right)$ of $t$ (modulo a renaming of the variable $\left.x_{1}, . ., x_{i-1}, x_{1}^{\prime}, \ldots, x_{r}^{\prime}, x_{i+1}, . ., x_{n}\right)$.

Conversely, an analogous proof is developed.
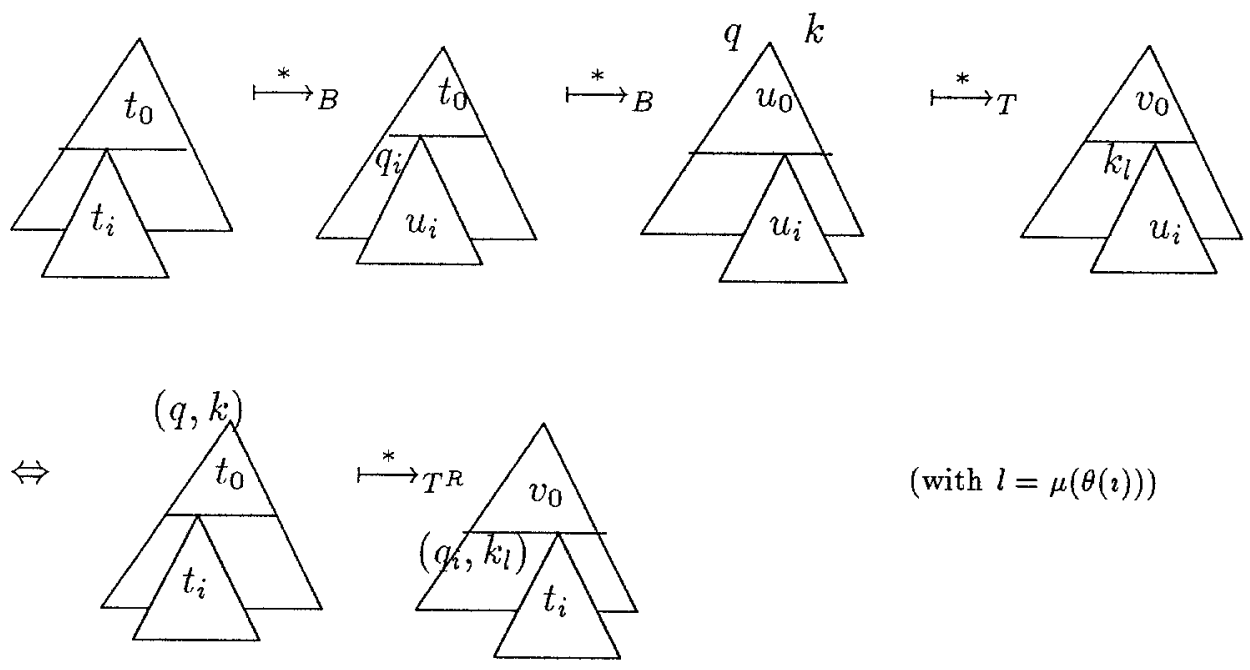

(with $l=\mu(\theta(2))$ ) 